\title{
Plasma Protein Binding as an Optimizable Parameter for Acidic Drugs $^{\text {霖 }}$
}

\author{
Philip Gardiner, Rhona J. Cox, and Ken Grime \\ Clinical Pharmacology \& Safety Sciences, Medicinal Chemistry and DMPK, Respiratory, Inflammation and Autoimmune (RIA), \\ R\&D BioPharmaceuticals, AstraZeneca, Gothenburg, Sweden
}

Received March 20, 2019; accepted May 17, 2019

\section{ABSTRACT}

The low volume of distribution associated with acidic molecules means that clearance (CL) must also be very low to achieve an effective half-life commensurate with once or twice daily dosing. Plasma protein binding (PPB) should not usually be considered a parameter for optimization, but in the particular case of acidic molecules, raising the PPB above a certain level can result in distribution volume becoming a constant low value equal to the distribution volume of albumin while acting to reduce $C L$ through restricting hepatic and renal access of unbound drug. Thus effective half-life can be increased. Here we detail the approaches and lessons learned at AstraZeneca during the optimization of acidic CXC chemokine receptor 2 (CXCR2) antagonists for the oral drug treatment of inflammatory diseases, resulting in discovery and clinical testing of $N$-[2-[(2,3-difluorophenyl)methylsulfanyl]6-[(2R,3S)-3,4-dihydroxybutan-2-yl]oxypyrimidin-4-yl]azetidine1-sulfonamide (AZD5069) and AZD4721, orally bioavailable acidic molecules with PPB of $<1 \%$, human hepatocyte intrinsic clearance values $<5 \mu \mathrm{l} / \mathrm{min}$ per $10^{6}$ cells and predicted human volume of distribution at steady state $\left(V_{\mathrm{ss}}\right)<0.3 \mathrm{l} / \mathrm{kg}$, resulting in effective half-lives in humans of 4 and 17 hours, respectively.

\section{SIGNIFICANCE STATEMENT}

Provided that the pharmacologic potency is high enough, modulation of plasma protein binding can form part of a viable strategy in drug discovery to optimize the effective half-life of drug candidates in humans.

\section{Introduction}

The delivery of compounds into clinical drug development with optimal pharmacokinetics (PK) is important to overall clinical success (Schuster et al., 2005; Cook et al., 2014); consequently, the ability to accurately predict human PK before clinical investment is of high importance (Di et al., 2013). Understanding relationships between pharmacological potency, drug property space, and PK, and being able to manipulate these through medicinal chemistry design, lies at the heart of modern drug discovery. This is itself reliant on detailed scrutiny of in vitro and in vivo drug metabolism and pharmacokinetic data and an awareness of associated caveats (Lavé et al., 2009; Di et al., 2013; Houston, 2013).

For acidic compounds the optimization of PK required for once or twice daily clinical dosing is particularly challenging. The majority of acidic drugs have low steady-state distribution volumes $\left(V_{\mathrm{ss}}\right)$ in the range of $0.1-0.31 / \mathrm{kg}$ (Grime et al., 2013) due to a combination of high plasma protein binding (PPB) and low tissue distribution (Smith et al., 2010) unless active hepatic uptake is a determining factor (Grover and Benet, 2009; Gardiner and Paine, 2011). Drug clearance

https://doi.org/10.1124/dmd.119.087163.

S This article has supplemental material available at dmd.aspetjournals.org. must therefore also be very low to achieve a suitable effective half-life (Smith et al., 2010; Bonn et al., 2016). Although PPB should generally not be a parameter for optimization in drug discovery projects (Smith et al., 2010), in the particular case of acidic molecules this can be an acceptable strategy. Raising the PPB can result in $V_{\mathrm{ss}}$ reaching the lower limiting value of approximately $0.1 \mathrm{l} / \mathrm{kg}$, the distribution volume of albumin (Rowland and Tozer, 1989); therefore, further PPB increases cannot result in lowering of $V_{\mathrm{ss}}$ below this value but can reduce clearance (CL) through restricting access of unbound drug to the hepatocytes. Through impacting CL but not $V_{\mathrm{ss}}$, half-life is increased and $C_{\max }$ (and therefore dose) is reduced if the aim is to maintain free drug concentrations above a fixed minimum effective drug concentration. This strategy for optimizing acidic drugs can work if there is excellent pharmacological potency allowing efficacious free blood levels to be maintained at target receptors (Grime and Riley, 2006). With these concepts in mind it is interesting to note that in an earlier review of the relevant literature we found that, of the nine examples of marketed oral acidic drugs with half-lives of more than 8 hours, seven of these have plasma protein binding of $\geq 99 \%$ (naproxen, piroxicam, atovaquone, diflunisal, cetirizine, warfarin, and oxaprozin; Grime et al., 2013).

The CXC chemokine receptor 2 (CXCR2) is expressed on the surface of neutrophils and is involved in the recruitment of these phagocytic

ABBREVIATIONS: AUC, area under the concentration-time curve; AZD4721, $N$-(6-\{[(2R,3S)-3,4-dihydroxy-2-butanyl]oxy\}-2-[(4-fluorobenzyl) sulfanyl]-4-pyrimidinyl)-3-methyl-1-azetidinesulfonamide; AZD5069, N-[2-[(2,3-difluorophenyl)methylsulfanyl]-6-[(2R,3S)-3,4-dihydroxybutan-2-yl]oxypyrimidin-4-yl]azetidine-1-sulfonamide; AZD8309, 5-[(2,3-difluorobenzyl)sulfanyl]-7-\{[(2R)-1-hydroxy-2-propanyl]amino\}[1,3]thiazolo[4,5-d]pyrimidin-

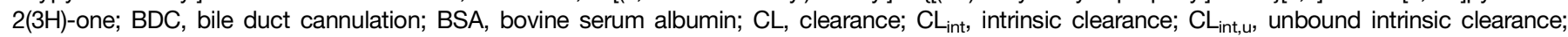
CXCR2, CXC chemokine receptor 2; $f_{u, i n c}$, fraction unbound in the incubation; HPLC, high-pressure liquid chromatography; HPMC, hydroxypropyl methylcellulose; IVIVE, in vitro-in vivo extrapolation; LC-MS/MS, liquid chromatography with tandem mass spectrometry; PO, by mouth; PPB, plasma protein binding; PK, pharmacokinetics; $V_{s s}$, volume of distribution at steady state; $V_{s s}$, v volume of distribution at steady state corrected for plasma protein binding. 
white blood cells to sites of inflammation through CXCL8 (interleukin-8, IL-8) signaling (Mukaida, 2003). Accordingly, antagonism of CXCR2 has been proposed as a strategy for the treatment of inflammatory diseases such as arthritis, chronic obstructive pulmonary disease, and cancer (Holmes et al., 1991; Murphy and Tiffany, 1991; Beeh et al., 2003; Jamieson et al., 2012; Highfill et al., 2014; Steele et al., 2016).

In the AstraZeneca CXCR2 drug discovery program, the acidity of the molecules was shown to be an important parameter for increasing both CXCR2 receptor binding and PPB (Austin et al., 2015). We outline the approaches taken and lessons learned from early CXCR2 clinical drug candidates and describe the preclinical data analysis that led to a strategy for achieving PK half-lives commensurate with once and twice daily dosing, ultimately resulting in the improved clinical drug candidates $N$-(2-[(2,3-difluorobenzyl)sulfanyl]-6-\{[(2R,3S)-3,4dihydroxy-2-butanyl]oxy \}-4-pyrimidinyl)-1-azetidinesulfonamide (AZD5069) and AZD4721.

\section{Materials and Methods}

\section{Chemicals}

All chemicals and reagents used were of the highest available grade. The AstraZeneca compounds including AZD5069 and AZD4721 were synthesized in-house by AstraZeneca (Austin et al., 2000; Willis et al., 2001; Bonnert, 2004; Ebden et al., 2004; Brough and McInally, 2005; Cheshire et al., 2006; Connolly et al., 2013). All other chemicals were purchased from Sigma-Aldrich (Poole, Dorset, United Kingdom). Hepatocyte suspension buffer was prepared from powder-equivalent Dulbecco's modified Eagle's medium (Sigma, Gillingham, United Kingdom) containing sodium HEPES ( $2.34 \mathrm{~g})$ and D-fructose $(0.4 \mathrm{~g})$ in 1 liter of Milli-Q water. The $\mathrm{pH}$ was adjusted to be 7.4 with $\mathrm{HCl}(1 \mathrm{M})$ at $37^{\circ} \mathrm{C}$. The hepatocyte suspension buffer contained bovine serum albumin (BSA, $2 \mathrm{~g}$ ) to wash cells after the hepatic isolation procedure, but intrinsic clearance incubation buffer was prepared without BSA.

\section{Hepatocyte Preparations}

Rat hepatocytes were isolated from male Sprague-Dawley rats using the two-step in situ collagenase perfusion method of Seglen (1976), as described elsewhere (Soars et al., 2007). Dog hepatocytes were isolated in house from male beagle dogs of approximately 1 year old. The isolation procedure was based on the two-step in situ collagenase perfusion method described in more detail elsewhere (McGinnity et al., 2004). Rat and dog hepatocytes were resuspended for assay use in suspension buffer without BSA, and their viability was assessed using the trypan blue exclusion method. Only cells with a viability $>80 \%$ were used. Cryopreserved isolated human hepatocytes were obtained from CellzDirect (Durham, NC) and thawed according to the supplier's instructions.

\section{Equilibrium Dialysis Measurement of Plasma Protein Binding}

Equilibrium dialysis measurement of PPB was performed as described elsewhere (Fessey et al., 2006). In brief, to one compartment of each of the dialysis cells were added $1 \mathrm{ml}$ of plasma and $10 \mu \mathrm{l}$ of a solution of compound at a concentration of $2 \mathrm{mM}$ in DMSO. The second section of each dialysis cell

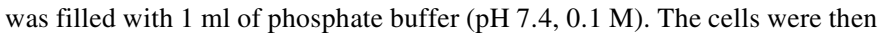
sealed, attached to the Dianorm unit, and rotated in a water bath at $37^{\circ} \mathrm{C}$ for 18 hours. Plasma $(100 \mu \mathrm{l})$ from the dialysis cell was added to phosphate buffer $(500 \mu \mathrm{l})$, and $500 \mu \mathrm{l}$ of the buffer solution from the dialysis cell was pipetted in to blank plasma $(100 \mu \mathrm{l})$.

The samples were analyzed using high-pressure liquid chromatography (HPLC) with MS detection. Due to the frequent nonlinear response of the mass spectrometry detectors, a calibration curve was obtained from dilutions of the stock solutions with 6-fold diluted plasma. The concentrations of the four standards were $0.05,0.15,0.5$, and $2.5 \mu \mathrm{M}$, and they injected in this order followed by the buffer samples and then the plasma samples.

The extent of PPB was calculated as described by Fessey et al. (2006). The chromatogram peak areas were processed automatically to use the calibration curve for each compound to calculate the concentration of unknowns.
These "concentrations" were corrected for differences in injection volumes and the dilution of the plasma. The percentage bound value was calculated using the following equation:

$$
\% \text { Bound }=100-100\left(\frac{1.2\left(\frac{\text { Buffer concentration } \times \text { Standard injection volume }}{\text { Buffer injection volume }}\right)}{6\left(\frac{\text { Plasma concentration } \times \text { Standard injection volume }}{\text { Plasma injection volume }}\right)}\right)
$$

The factor of 1.2 in the numerator accounts for the small dilution of the aqueous samples with plasma. The factor of 6 in the denominator serves to correct for the 6-fold dilution of the plasma samples with buffer.

Each compound was measured in duplicate in the same experiment. The precision of the assay was assessed over many duplicate measurements performed across structurally diverse compounds. Table 1 contains the ratio of the percent free for duplicate pairs of measurements from the same batch of plasma for 1681 compounds measured in the protein binding assay. The distribution of duplicate pair-ratios was almost normally distributed; applying the assumption of normality, it can then be calculated that $95 \%$ of duplicate ratios are less than 2.14 . Any duplicate ratio larger than 2.14 is thus a rare occurrence and can be termed an outlier, and the assay would be repeated for this compound.

Table 2 contains mean free percent ratios when the same compound was measured on different days - that is, with a different batch of plasma. The mean percent free on 1 day (mean of two data points) was then compared with the mean percent free on another day for each compound.

The data in Table 2 show that the average ratio of the mean percent free determined 1 day to the mean percent free determined on another day is about 1.5 , with a S.D. of about 0.5 . The standard deviations in Table 2 are not directly comparable with the standard deviations in Table 1 because the standard deviations in Table 2 are the average S.D. of the ratio of two means (each a mean of two data points), whereas the standard deviations in Table 1 are the average standard deviation of the ratio of two single measurements of the percent free.

The 95\% limit for duplicate ratios in Table 2 indicates that if a compound is measured on two different days and the mean percent free differs by a factor of greater than about 2.6, then the data must be viewed as suspect (even if each of the two duplicates has a satisfactory duplicate ratio), and more experiments should be performed. Hence, during the discovery phase when comparing the percent free of different compounds, the assay performance analysis indicated that for compounds measured once (in duplicate), a difference between compounds $\geq 2.6$ in the fraction unbound $\left(f_{\mathrm{u}}\right)$ was required to be judged a significantly different percent free value at the $95 \%$ level. In general, compounds were tested on two separate occasions and key compounds such as AZD5069 and AZD4721 on five or more occasions.

\section{Determination of Blood-Plasma Ratio}

Stock concentrations of test compounds were 100-fold above final incubation concentrations, and these were pipetted into fresh blood and plasma $(0.5 \mathrm{ml})$. After 15 minutes of incubation $\left(37^{\circ} \mathrm{C}\right)$ and centrifugation $(9000 \mathrm{~g}, 4$ minutes) using an MSE MicroCentaur centrifuge (Fisher Scientific, Loughborough, United Kingdom), aliquots from both the blood and plasma incubations were pipetted into methanol and stored at $-20^{\circ} \mathrm{C}$ for 1 hour. Samples were centrifuged $(2000 \mathrm{~g}$, 20 minutes), and the supernatant was analyzed by liquid chromatography with tandem mass spectrometry (LC-MS/MS). The blood-plasma ratio was calculated from the ratio of the analyte concentration in directly spiked plasma to that in plasma isolated from spiked blood. Key compounds such as AZD5069 and AZD4721 were tested across species.

\section{Measurement of $\log \mathrm{D}_{7.4}, \mathrm{p} K_{\mathrm{a}}$, and Solubility}

For $\log \mathrm{D}_{7.4}$ determination, separation of compounds between 1-octanol and phosphate buffer $\left(0.02 \mathrm{M}, \mathrm{pH} 7.4\right.$ at $\left.20^{\circ} \mathrm{C}\right)$ was determined using a shake flask method. Octanol and aqueous tiers were analyzed by LC-MS/MS as described here. The $\mathrm{p} K_{\mathrm{a}}$ was measured using a Sirius GLpKa instrument with DPAS (Dip Probe Absorption Spectroscopy) attachment (Sirius Analytical Instruments Ltd, Forest Row, United Kingdom). Samples of test compounds were placed in vials in a movable autosampler tray for titration. Assays were set up using the GlpKaControl software, and the results were analyzed using the pKaLOGP and $\mathrm{pKaUV}$ software, which allows determination of multiple $\mathrm{p} K_{\mathrm{a}}$ values using complex curve fitting analyses. 
TABLE 1

Mean, S.D., and 95\% limit for pairs of mean percent free data in human, rat, and dog plasma binding data in the same batch of plasma

\begin{tabular}{lcccr}
\hline Species & Mean Duplicate Ratio & S.D. in Duplicate Ratio & 95\% of Duplicate Ratios Below & $n$ \\
\hline Human & 1.35 & 0.48 & 2.14 & 1077 \\
Rat & 1.25 & 0.30 & 1.74 & 356 \\
Dog & 1.26 & 0.46 & 1.97 & 248 \\
\hline
\end{tabular}

Solubility was measured as described elsewhere (Wenlock et al., 2011b). In brief, compounds $(1 \mathrm{mg})$ were placed into separate 2-ml glass tubes, and phosphate buffer $(\mathrm{pH} 7.4,0.1 \mathrm{M}, 1 \mathrm{ml})$ was added. The vials were shaken for a minimum of 18 hours. After shaking, the saturated solutions were transferred to 2 -ml centrifuge tubes and were centrifuged at $13,000 \mathrm{~g}$ for 15 minutes. The supernatants were then removed, placed into new centrifuge tubes, and centrifuged again at $13,000 \mathrm{~g}$ for 15 minutes.

Aliquots of the supernatants from the second centrifugation were analyzed by LC-MS/MS along with standard concentration stock solutions, prepared by adding DMSO (800 $\mu \mathrm{l}$ ) to compound (1 mg) and sonicating (15 minutes). Single measures were generally regarded as sufficient except for key compounds such as AZD5069 and AZD4721.

\section{Determination of Intrinsic Clearance}

Test compound stock solutions were prepared in DMSO at 100-fold incubation concentration $(100 \mu \mathrm{M})$. Stock solution $(10 \mu \mathrm{l})$ was added to the hepatocyte suspension buffer $(490 \mu \mathrm{l})$. This solution and separately the hepatocytes ( 2 million viable cells $/ \mathrm{ml}$ ) were preincubated for 5 minutes in a shaking ( 80 oscillations $/ \mathrm{min}$ ) waterbath at $37^{\circ} \mathrm{C}$. Reactions were initiated by adding hepatocyte suspension $(500 \mu \mathrm{l})$ to test compound solution giving a final substrate concentration of $1 \mu \mathrm{M}$ and $1 \%(\mathrm{v} / \mathrm{v})$ DMSO. Aliquots ( $40 \mu \mathrm{l}$ ) were removed at $0,2,6,15,30,45,60$, and 90 minutes, and the reactions were quenched in $120 \mu \mathrm{l}$ of ice-cold methanol. Samples were subsequently frozen for 1 hour at $-20^{\circ} \mathrm{C}$ and then centrifuged at $2000 \mathrm{~g}$ for 20 minutes at $4^{\circ} \mathrm{C}$.

The supernatants were removed for LCMS/MS analysis. Analytical LC-MS/ MS peak areas of samples were $\log _{\mathrm{e}}$-transformed, and elimination rate constants $k\left(\min ^{-1}\right)$ were derived from the slopes of the $\log _{\mathrm{e}}$ [substrate]-time plots. Intrinsic clearance was calculated from $\mathrm{CL}_{\text {int }}=k \times V$, where $V$ represents the incubation volume ( $\mathrm{ml} / \mathrm{mg}$ protein or $\mathrm{ml} / \mathrm{million}$ cells). Key compounds such as AZD5069 and AZD4721 were incubated on at least three separate occasions in rat, dog, and human hepatocytes.

\section{Methods for Prediction of Hepatic Metabolic CL}

Clearance predictions were made using a "regression line approach," whereby an existing in vitro-in vivo unbound $\mathrm{CL}_{\mathrm{int}}$ data set (for which in vivo $\mathrm{CL}_{\mathrm{int}}$ values represent metabolic clearance only) is used as a framework for predicting the in vivo clearance for novel compounds (Sohlenius-Sternbeck et al., 2012). However, determination of whether the clearance of a compound is well predicted from the in vitro data comes from the assessment of $\mathrm{CL}_{\text {int,u,in vivo }} / \mathrm{CL}_{\text {int,u,in vitro, }}$ which should be between 0.5 and 2.0 (Grime et al., 2013). See Table 3 for AZD5069 and AZD4721 predictions.

\section{Preclinical In Vivo Studies}

All in vivo work was subject to internal ethical review and conducted in accordance with UK Home Office requirements under the Animals Scientific Procedures Act (1986). Healthy virus-antibody-free male Sprague-Dawley rats were obtained from Charles River (Margate, United Kingdom). They were housed in a light-controlled room $\left(19 \pm 2{ }^{\circ} \mathrm{C}\right.$ and $55 \% \pm 10 \%$ humidity $)$. A Teklad 2021 diet (Harlan) was used with drinking water ad libitum. In-house bred male beagles (housed in pairs, temperature of $18 \pm 2{ }^{\circ} \mathrm{C}$, and humidity $55 \% \pm 10 \%$ ) were fed with a SDS D3 (E) Dog Maintenance diet with water ad libitum.

\section{Intravenous and Oral Pharmacokinetic Studies}

Rat. AZD5069 and AZD4721 were dosed at $1 \mathrm{mg} / \mathrm{kg}$ as a bolus to the tail vein in DMA: $\mathrm{H}_{2} \mathrm{O}(1: 9 \mathrm{v} / \mathrm{v})$ to conscious male rats $(n=3$ or 4$)$. Doses by mouth (PO) were given via oral gavage in a Tween $(0.1 \%)$ hydroxypropyl methylcellulose (HPMC) $(1 \%)$ suspension $(n=3)$. Serial blood samples (200-300 $\mu$ l) were taken, and the plasma was recovered after centrifugation.

Dog. AZD5069 and AZD4721 were dissolved in bicarbonate buffer ( $\mathrm{pH} 10.0)$ including $10 \%$ ethanol to a concentration of $1 \mathrm{mg} / \mathrm{ml}$. The dose was given via infusion (30 minutes) to the cephalic vein. PO doses were given via oral gavage in a Tween $(0.1 \%)$ HPMC $(1 \%)$ suspension $(n=2)$. Serial blood samples $(2.5 \mathrm{ml})$ were taken and centrifuged to obtain plasma.

\section{Intravenous Bile Duct Cannulation Studies (AZD5069 and AZD4721)}

Rat. After at least 1 week of acclimatization, rats (250-350 g) were surgically prepared under isoflurane anesthesia. The bile duct was cannulated with a bilepancreatic catheter (constructed by polyethylene catheter Physiocath and P90; Data Sciences International, St. Paul, MN). An opening was made in the duodenum, and the catheter was inserted for bile recirculation. A small cut in the skin (around the area of the scapula) facilitated subcutaneous routing of the catheter. After 3 days' recovery, the animals were connected to a swivel system (Instech Laboratories, Plymouth Meeting, PA) that allowed freedom of movement during the experiment. Cannulae were inserted into the jugular and carotid blood vessels for dosing and blood sampling respectively.

A bile sample was taken over the first hour after dosing and then every halfhour until 7 hours. Blood samples (200-300 $\mu$ l) were taken at the midpoint of the bile collections, and the plasma was prepared as stated earlier. Urine was also collected, and all samples were stored at $-20^{\circ} \mathrm{C}$.

Dog. After at least 4 weeks of acclimatization, a bile duct cannulation (BDC) operation was performed on two dogs following the technique described by Kissinger et al. (1998). A 1-month recovery period followed before the study. AZD5069 and AZD4721 were dissolved in bicarbonate buffer (pH 10.0) including 10\% ethanol to a concentration of $1 \mathrm{mg} / \mathrm{ml}$. The dose was given via infusion (30 minutes) to the cephalic vein. Jugular vein sampling of blood $(2.5 \mathrm{ml})$ was made into EDTA-containing tubes at 0,15 , $30,60,120,180,300,420,720$, and 1440 minutes after the start of the infusion before centrifugation $(1110 \mathrm{~g}, 10$ minutes) for plasma collection. Bile and urine collection continued for 24 hours. All samples were stored at $-20^{\circ} \mathrm{C}$ before analysis.

Sample Preparation. Before analysis, samples from the in vivo studies were prepared as follows: Aliquots $(50 \mu \mathrm{l})$ of plasma were added to methanol $(150 \mu \mathrm{l})$.

TABLE 2

Mean, S.D., and $95 \%$ limit for pairs of mean percent free data in human, rat, and dog plasma binding data in different batches of plasma

\begin{tabular}{lcccr}
\hline Species & Mean Duplicate Ratio & S.D. in Duplicate Ratio & $95 \%$ of Duplicate Ratios Below & $n$ \\
\hline Human & 1.60 & 0.61 & 2.60 & 161 \\
Rat & 1.33 & 0.49 & 2.13 & 58 \\
Dog & 1.55 & 0.46 & 2.30 & 71 \\
\hline
\end{tabular}


TABLE 3

Preclinical data summary and predicted human PK for AZD5069 and AZD4721

\begin{tabular}{|c|c|c|c|c|c|c|}
\hline & \multicolumn{3}{|c|}{ AZD5069 } & \multicolumn{3}{|c|}{ AZD4721 } \\
\hline $\begin{array}{l}\text { CXCR2 human IL- } 8 \text { binding FMAT } \mathrm{pIC}_{50}{ }^{a} \\
\text { Hepatocyte } \mathrm{CL}_{\text {int }}\left(\mu 1 / \text { min per } 10^{6} \text { cells }\right) \text { rat } / \mathrm{dog} / \text { human } \\
\text { Solubility, } \mu \mathrm{M} \\
\mathrm{LogD}_{7.4} / \mathrm{p} K_{\mathrm{a}} \text { per calculated } f_{\mathrm{u} \text {,inc }} \\
\mathrm{PPB}(\% \text { free }) \text { rat } / \mathrm{dog} \text { per human } \\
\text { Blood-plasma ratio rat } / \text { dog per human }\end{array}$ & \multicolumn{3}{|c|}{$\begin{array}{c}8.5 \\
12 / 11 / 4\left(\mathrm{HLM} \mathrm{CL}_{\text {int }}=14\right) \\
190 \\
1.7 / 5.8 / 0.8 \\
2.4 / 2.3 / 0.59 \\
0.6 / 0.6 / 0.6\end{array}$} & \multicolumn{3}{|c|}{$\begin{array}{c}8.4 \\
10 / 4 / 3\left(\mathrm{HLM} \mathrm{CL}_{\text {int }}\right. \\
97 \\
1.9 / 5.7 / 0.8 \\
0.42 / 0.46 / 0.11 \\
0.8 / 0.7 / 0.6\end{array}$} \\
\hline In Vivo PK & Rat & Dog & Human Prediction & Rat & Dog & Human Prediction \\
\hline Predicted hepatic metabolic CL (ml/min per kilogram) & 5.4 & 5.9 & 0.36 & 1.7 & 0.80 & 0.04 \\
\hline Observed CL (ml/min per kilogram) & 4.2 & 6.5 & & 2.4 & 0.50 & \\
\hline $\mathrm{CL}_{\text {renal }}(\mathrm{ml} / \mathrm{min}$ per kilogram $)$ & $<0.01$ & $<0.01$ & & $<0.01$ & $<0.01$ & \\
\hline $\mathrm{CL}_{\text {biliary }}(\mathrm{ml} / \mathrm{min}$ per kilogram $)$ & 0.10 & $0.10^{b}$ & & $<0.01$ & 0.04 & \\
\hline In vivo/in vitro unbound $\mathrm{CL}_{\mathrm{int}}{ }^{c}$ & 0.76 & 1.2 & & 1.4 & 0.6 & \\
\hline$V_{\mathrm{ss}}(1 / \mathrm{kg})$ & 0.32 & 1.1 & 0.25 & 0.19 & 0.15 & 0.11 \\
\hline$t_{1 / 2}(\mathrm{~h})(\mathrm{PO})$ & $\begin{array}{c}1.0 \\
(2.5)\end{array}$ & $\begin{array}{l}0.44 \\
(2.6)\end{array}$ & 7.5 & $\begin{array}{c}1.3 \\
(2.7)\end{array}$ & $\begin{array}{c}3.7 \\
(8.4)\end{array}$ & 30 \\
\hline$F(\%)$ & 31 & 48 & & 45 & 82 & \\
\hline
\end{tabular}

${ }^{a}$ Ligand binding assay described in Connolly et al. (2013).

${ }^{b}$ The dog BDC study was performed after candidate drug selection. A summary of key preclinical in vitro and in vivo data are shown in Table 3 . Based on IVIVE and negligible renal and biliary clearance in rat and negligible dog renal clearance (at the time of proceeding to phase 1 clinical studies, no bile duct cannulated dog PK study had been performed on AZD5069), human CL was predicted to be $0.36 \mathrm{ml} / \mathrm{min}$ per kilogram (driven by entirely hepatic metabolism), $V_{\mathrm{ss}}$ was predicted to be 0.25 $1 / \mathrm{kg}$ (based on rat and dog unbound $V_{\mathrm{ss}}$ measured in non-bile duct cannulated animals), and effective half-life was consequently predicted to be 7.5 hours for AZD5069. ${ }^{c}$ In vivo $\mathrm{CL}_{\mathrm{int}, \mathrm{u}}$ calculated from the well-stirred liver model (Pang and Rowland, 1977) as follows: $\mathrm{CL}_{\mathrm{int}, \mathrm{u}}=\mathrm{CL}_{\mathrm{H}} / f_{\mathrm{u}, \mathrm{b}} \times\left(1-\mathrm{CL}_{\mathrm{H}} / \mathrm{Q}_{\mathrm{H}}\right)$ and in vitro $\mathrm{CL}_{\mathrm{int}, \mathrm{u}}$ calculated as $\mathrm{CL}_{\mathrm{int}} / f_{\mathrm{u}, \mathrm{inc}}$.

Water was added to the bile and urine before analysis. Standard curves and quality control (QC samples) were made up in matrix blanks.

\section{Sample Analysis}

Samples were analyzed by LC-MS/MS (HP1100 HPLC system supplied by Hewlett Packard, Quattro Ultima mass spectrometer supplied by Micromass; Waters, Milford, MA). Electrospray ionization mode was used. For HPLC, a Waters Symmetry C8 $3.5 \mu \mathrm{m}(2.1 \times 30 \mathrm{~mm})$ column was used, injecting $10 \mu \mathrm{l}$ of each sample. The mobile phase was water plus $0.1 \%$ formic acid and methanol plus $0.1 \%$ formic acid.

\section{Pharmacokinetics}

Parameters (clearance, $V_{\mathrm{ss}}$, and terminal half-life) were derived from the concentration-time profile by noncompartmental analysis using WinNonlin Professional Version 5.2 (Pharsight Corporation, Mountain View, CA). Clearance estimated as dose divided by AUC, where AUC is the area under the concentration-time curve from time zero extrapolated to infinity. AUC is estimated by $\mathrm{AUC}(0-$ last $)+C_{\text {last }} / \lambda \mathrm{z}$, where $C_{\text {last }}$ is the last observed quantifiable concentration. $V_{\mathrm{ss}}$ was estimated by dividing the mean residence time by the clearance. Terminal half-life was estimated as $(\ln 2) / \lambda z$, where $\lambda z$ is the terminal rate constant, estimated by log-linear least squares regression of the terminal part of the concentration-time curve.

\section{Prediction of Human $V_{\text {ss }}$ and Effective Half-Life}

The average of rat and $\operatorname{dog} V_{\mathrm{ss}, \mathrm{u}}\left(V_{\mathrm{ss}}\right.$ adjusted for PPB) was used to predict human $V_{\mathrm{ss}, \mathrm{u}}$, which was then multiplied by human PPB to predict human $V_{\mathrm{ss}}$. Human effective half-life was predicted from $0.693 \times$ Predicted $V_{\text {ss }} /$ Predicted CL.

\section{Metabolite Identification Studies}

In vitro incubations were performed with isolated rat, dog, and human hepatocytes (2 million cells/ml). AZD5069 and AZD4721 $(10 \mu \mathrm{M})$ were incubated for 1 hour at $37^{\circ} \mathrm{C}$. The incubations were quenched with two volumes of methanol, vortexed, and stored at $-20^{\circ} \mathrm{C}$. Samples were prepared just before MS analysis by centrifugation at $9000 \mathrm{~g}$ for 30 minutes and by transferring the supernatant to sample vials. In vivo samples were prepared as follows: urine and bile samples were diluted one part bile or urine to one part methanol, vortexed, and frozen. Plasma samples were quenched with three volumes of methanol and frozen. Samples were defrosted, vortexed, and centrifuged (9000 $\mathrm{g}$ for 30 minutes) just before analysis, and the supernatant was taken and diluted 1:1 with water.

Samples were analyzed in positive ion mode. The MS method used consisted of two MS channels, a normal MS scan and a source induced decomposition (SID) channel to provide structural data on parent compound and metabolites. Where necessary, MS/MS was also performed. All MS data were acquired with a mass accuracy of $5 \mathrm{ppm}$. After the analysis, the data were processed manually to identify the major metabolites based on MS response. Minor metabolites were defined as representing $\leq 1 \%$ of the total response.

\section{Clinical PK}

Both the AZD5069 and AZD4721 studies were approved by an independent ethics committee before initiation and were performed in accordance with the ethical principles that have their origin in the Declaration of Helsinki and are consistent with International Conference on Harmonization/Good Clinical Practice, applicable regulatory requirements, and the AstraZeneca Policy on Bioethics. Informed consent was obtained from all volunteers before initiation of the studies. AZD5069 human pharmacokinetic data were generated in a phase 1, blinded, randomized, placebo-controlled, single-center study (NCT00953888 and Cullberg et al., 2018) to investigate the safety, tolerability, pharmacokinetics, and pharmacodynamics of oral AZD5069 after single ascending doses in Caucasian male and female healthy volunteers with body mass indices between 18 and $30 \mathrm{~kg} / \mathrm{m}^{2}$ (inclusive) and weights between 50 and $100 \mathrm{~kg}$ (inclusive). The study was performed at AstraZeneca Clinical Pharmacology Unit, Queens Medical Centre (Nottingham, United Kingdom).

Serial blood samples were taken up to 96 hours after the dose administration, and plasma samples were analyzed using validated bioanalytical methods at AstraZeneca R\&D Charnwood. The PK parameters were derived using standard noncompartmental methods.

AZD4721 human pharmacokinetic data were generated in a separate phase 1, blinded, randomized, placebo-controlled, single-center study (NCT01889160) to investigate the safety, tolerability, pharmacokinetics, and pharmacodynamics of oral AZD4721 after single ascending doses in Caucasian male healthy volunteers with body mass indices between 18 and $30 \mathrm{~kg} / \mathrm{m}^{2}$ (inclusive) and weights between 50 and $100 \mathrm{~kg}$ (inclusive). The study was performed at Quintiles Drug Research Unit at Guy's Hospital London, England.

Serial blood samples were taken up to 216 hours after the dose administration, and plasma samples were analyzed using validated bioanalytical methods. The 
Thiazolone series<smiles>CC(CO)Nc1nc(SCc2cccc(F)c2F)nc2[nH]c(=O)sc12</smiles>

Sulfamide series<smiles>C[C@H](CO)Nc1cc(NS(=O)(=O)N2CCOCC2)nc(SCc2cccc(F)c2F)n1</smiles>

Early monocyclic sulfamide lead<smiles>C[C@H](Oc1cc(NS(=O)(=O)N2CCC2)nc(SCc2cccc(F)c2F)n1)C(O)CO</smiles>

AZD5069<smiles>CC1CN(S(=O)(=O)Nc2cc(O[C@H](C)C(O)CO)nc(SCc3ccc(F)cc3)n2)C1</smiles>

AZD4721

Fig. 1. Chemical development of CXCR2 antagonists from an early clinical candidate AZD8309, to AZD5069 and AZD4721 with twice-daily and once-daily dosing in humans, respectively.

pharmacokinetic parameters were derived using noncompartmental methods with WinNonlin Professional Version 5.2, or higher (Pharsight).

\section{Data Analysis}

Impact of PPB on Rat Pharmacokinetics. In vivo rat $C L$ was plotted against rat PPB data and separately rat $V_{\mathrm{ss}}$ for the thiazolone and sulfamide chemical series investigated in the CXCR2 program.

Simulating the Impact of PPB on Human Half-Life. The CL prediction method (using the regression line approach and unbound in vivo and in vitro $\mathrm{CL}_{\text {int }}$; see Materials and Methods) was used to calculate $\mathrm{CL}$ at various values of PPB when human hepatocyte $\mathrm{CL}_{\text {int }}$ was of $1 \mu \mathrm{l} / \mathrm{min}$ per million hepatocytes. Changes in half-life were calculated as $0.693 \times V_{\mathrm{ss}} / \mathrm{CL}$ when $V_{\mathrm{ss}}$ was constant at $0.21 / \mathrm{kg}$ (unaffected by plasma protein binding).

\section{Results}

\section{Analysis of the Impact of PPB on Rat Pharmacokinetics}

Plasma protein binding was shown to relate to rat clearance for two chemical series of compounds, thiazolones and sulfamides (Figs. 1 and 2A). Vss was found to be low, as expected for acidic molecules, with $70 \%$ of the molecules having Vss less than 0.4 1/kg (Fig. 2B). The data indicates the lack of a relationship between plasma protein binding and distribution volume.

\section{Simulating the Impact of PPB on Human Half-Life}

Based on the observations in Fig. 2, a simple simulation was performed assuming $V_{\mathrm{ss}}$ to be typically low for an acidic molecule and constant at $0.21 / \mathrm{kg}$. Assuming that it is possible to keep hepatic intrinsic clearance of unbound drug constant while increasing PPB, the prediction demonstrates that PPB influences half-life through reducing clearance and indicates that PPB in excess of $99.2 \%$ could achieve a half-life in excess of 15 hours (Fig. 3).

\section{Preclinical Data and Human PK Predictions}

A summary of key preclinical in vitro and in vivo data for AZD5069 and AZD4721 is shown in Table 3.

AZD5069. Analysis of rat PK data showed that bile duct cannulation had no discernible effect on the PK profile (Fig. 4). Subsequent to the readout of the clinical PK data for AZD5069, a BDC study revealed the dog PK profile to have a lower exposure and shorter observed terminal phase than from the noncannulated intravenous PK experiment, even though biliary clearance of parent AZD5069 was measured as $0.1 \mathrm{ml} / \mathrm{min}$ per kilogram $(1.4 \%$ of total $\mathrm{CL})$.

AZD4721. As with AZD5069, human CL was predicted to be driven entirely by hepatic metabolism. With lower human hepatocyte $\mathrm{CL}_{\mathrm{int}}$ and higher human PPB, the effective human half-life was predicted to be considerably longer than that predicted for AZD5069. Unlike AZD5069,
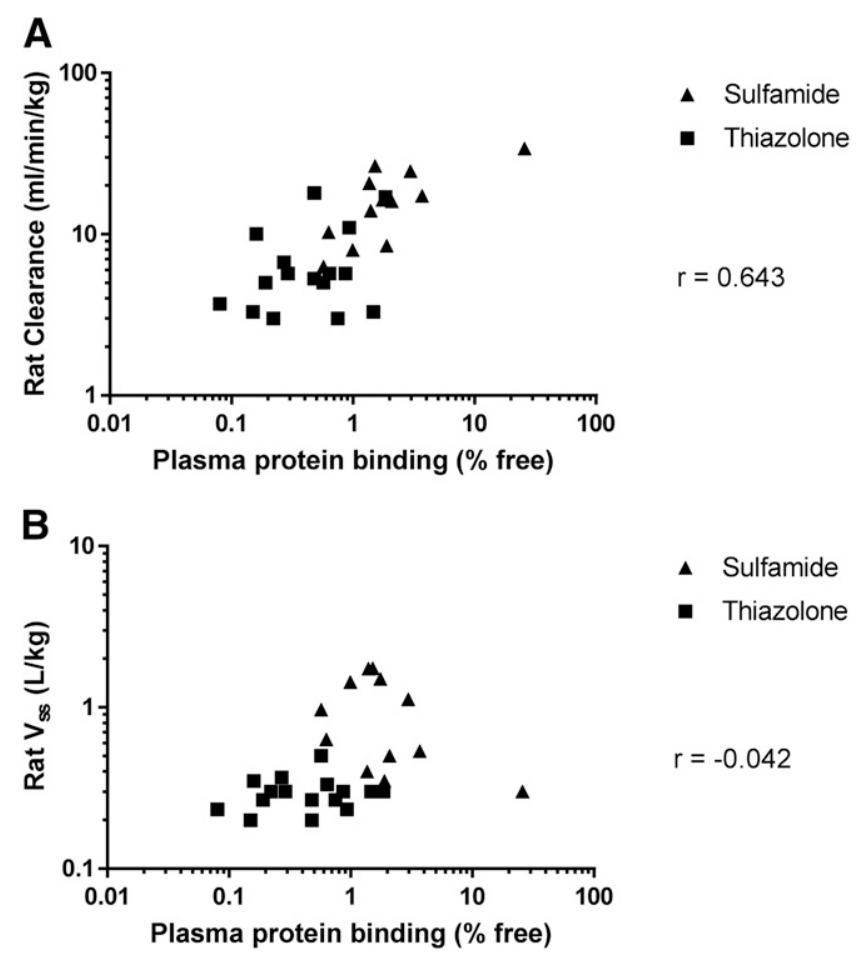

Fig. 2. The relationship between (A) plasma protein binding and rat plasma clearance and (B) rat steady-state volume of distribution $\left(V_{\mathrm{ss}}\right)$ for AstraZeneca CXCR2 antagonists from the sulfamide $(\boldsymbol{\Lambda})$ and thiazolone $(\boldsymbol{\square})$ chemical series with correlation statistics. Individual compound data, chemical series, and structure references are provided in the Supplemental Information (see Supplemental Table 1). 


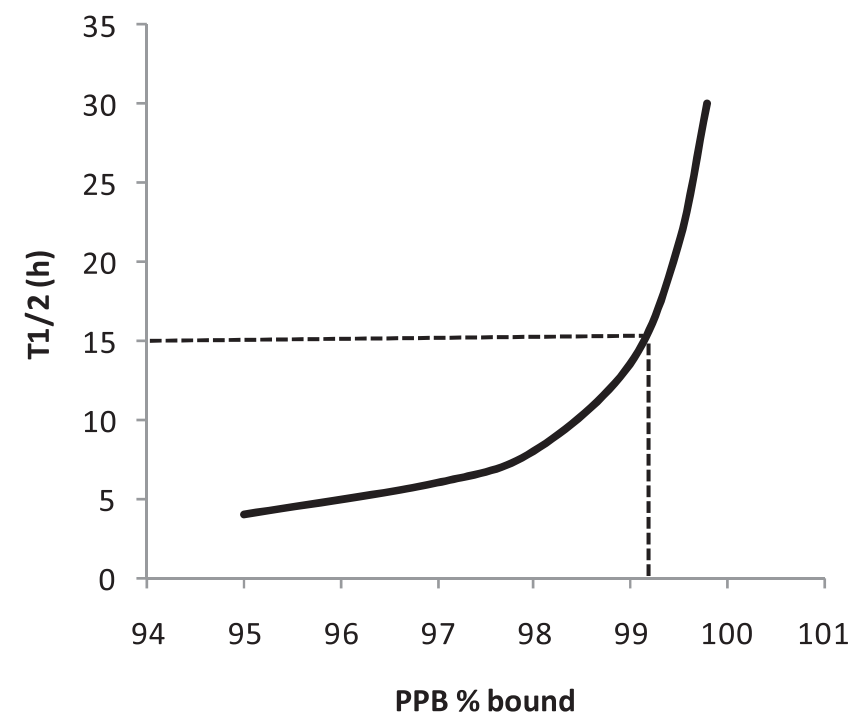

Fig. 3. Simulation highlighting the predicted effect of drug binding to plasma proteins on half-life $\left(0.693 \times V_{\mathrm{ss}} / \mathrm{CL}\right)$. CL was predicted using the regression line approach (Materials and Methods), with human hepatocyte $\mathrm{CL}_{\mathrm{int}}$ set to be $1 \mu \mathrm{l} / \mathrm{min}$ per million hepatocytes and $V_{\mathrm{ss}}$ kept constant at $0.2 \mathrm{l} / \mathrm{kg}$ (unaffected by plasma protein binding). Dotted line indicates PPB of $99.2 \%$ at $t_{1 / 2}$ of 15 hours.

dog intravenous and BDC PK profiles for AZD4721 were very similar in shape and absolute concentration throughout the sampled interval (Fig. 5).

\section{Biotransformation Data}

AZD5069. AZD5069 metabolism was complex, with multiple pathways observed in rats, dogs, and humans. However, the major pathways identified were direct glucuronide conjugation at the diol group, $S$-debenzylation (loss of the methylene-difluorophenyl moiety) and oxidations on the azetidine ring (Fig. 6). Qualitatively, the metabolites were generally similar in each of the species but there were quantitative differences, partly as a result of different metabolic turnover rates.

The most substantial difference was observed in dogs, with the $O$-glucuronide of AZD5069 being by far the most prominent metabolic route and representing $99 \%$ of MS response. Absolute configuration as an $O$-glucuronide on the 4-hydroxy position of the 3,4-dihydroxy-2yloxy side chain of AZD5069 was later determined by NMR (data not shown).

AZD4721. A similar metabolism pattern to AZD5069 was observed for AZD4721. However, azetidine oxidation products were more minor than for AZD5069, possibly due the addition of the methyl at the

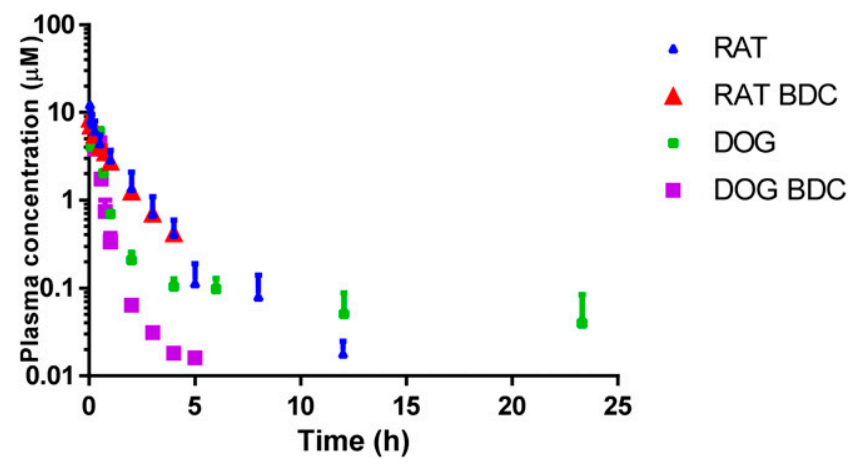

Fig. 4. AZD5069 plasma concentration-time profile after intravenous dosing ( $1 \mathrm{mg} /$ $\mathrm{kg}$ ) to bile duct cannulated (red triangles) and noncannulated (blue triangles) rats and bile duct cannulated (purple squares) and noncannulated (green squares) dogs.

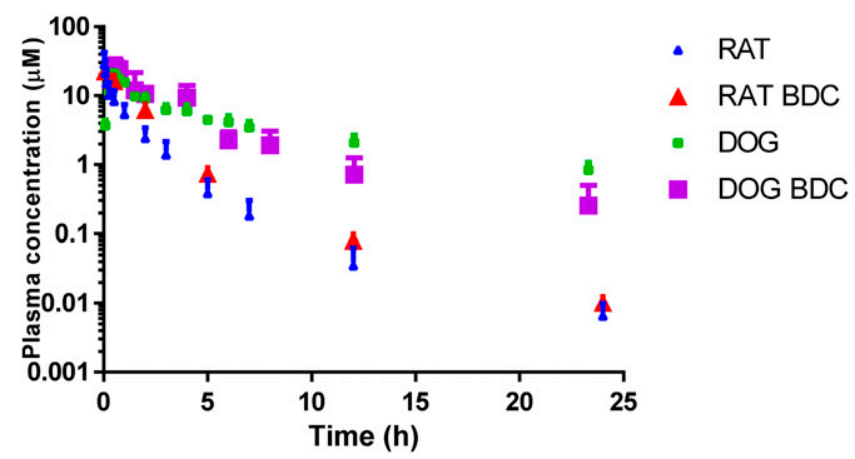

Fig. 5. AZD4721 plasma concentration-time profile after intravenous dosing ( $1 \mathrm{mg} / \mathrm{kg})$ to bile duct cannulated (red triangles) and noncannulated (blue triangles) rats and bile duct cannulated (purple squares) and noncannulated (green squares) dogs.

azetidine three-position as well as lower turnover rates in hepatocytes. As with AZD5069, the $O$-glucuronide was the major metabolite observed in dogs in vitro and in vivo; however, rather than representing $99 \%$ of the drug-related MS response observed for AZD5069, this was reduced to $56 \%$.

From incubations with human hepatocytes, formation of a carboxylic acid metabolite represented $4 \%$ of drug-related MS response. This was more major than in the either of the other two species or for that observed with AZD5069 (Fig. 6).

\section{Clinical PK}

AZD5069. Exposure of AZD5069 (AUC and $C_{\max }$ ) was found to be approximately dose proportional after dosing of $0.1-200 \mathrm{mg}$ with an effective half-life of 4 hours. The 24-hour plasma profile for the $120 \mathrm{mg}$ dose is shown in Fig. 7.

AZD4721. The geometric mean effective half-life of AZD4721 ranged from 14 to 19 hours across the dose range studied (19-730 mg). AUC and $C_{\max }$ increased in proportion to the dose. The 24-hour plasma profile for the 150-mg dose is shown in Fig. 7.

\section{Discussion}

The modulation of physicochemical properties to manipulate PK parameters lies at the heart of effective drug discovery (Riley et al., 2002; Davis and Riley, 2004; Di et al., 2013; Lombardo et al., 2014). Indeed, optimizing molecular properties such as size, lipophilicity, and polarity has been directly linked to greater success in clinical drug development (Waring et al., 2015).

Early CXCR2 antagonists in the AstraZeneca program had bicyclic thiazolone cores and were relatively lipophilic weak acids with poor solubility (Walters et al., 2008) but nonetheless their properties were sufficiently promising for the lead compound AZD8309 5-[(2,3-difluorobenzyl)sulfanyl]-7-\{[(2R)-1-hydroxy-2propanyl]amino $\}[1,3]$ thiazolo[4,5-d]pyrimidin-2(3H)-one; (Fig. 1) to be taken into the clinic. Although AZD8309 proved a valuable tool compound to investigate the role of CXCR2 antagonists in inflammatory lung disease (Virtala et al., 2012; Leaker et al., 2013) its PK properties, exemplified by variable human bioavailability and a short half-life, were regarded as unsuitable for further clinical development. In the main these molecules had high PPB, high in vitro metabolic instability and low incubational binding, which gave poor predictions of in vivo rat and $\operatorname{dog}$ CL when using a simplified version of the well-stirred model, ignoring drug-binding terms. These observations and those of others (Houston and Carlile, 1997; Iwatsubo et al., 1997; Carlile et al., 1999; Ito and Houston, 2005) gave us a greater appreciation of the impact of drug binding on CL predictions, and revised CL prediction models resulting 


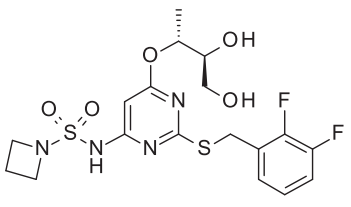

AZD5069<smiles>COOC(C)C(C)Oc1cc(NS(=O)(=O)N2CCC2)nc(SCc2cccc(F)c2F)n1</smiles>

glucuronidation

B

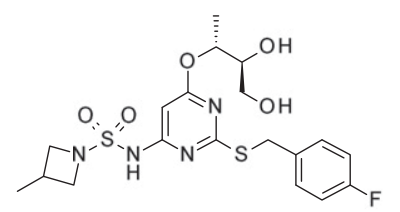

AZD4721

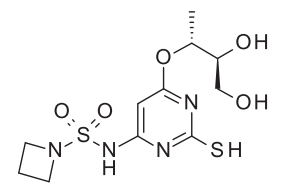

S-debenzylation<smiles>C[C@@H](Oc1cc(NS(=O)(=O)N2CC(O)C2O)nc(SCc2cccc(F)c2F)n1)C(O)CO</smiles>

azetidine oxidation

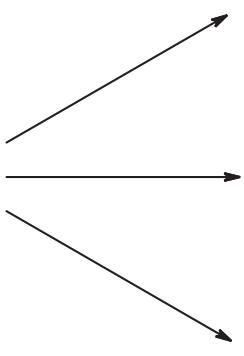

$$
\text { (-glucuronide }
$$

glucuronidation

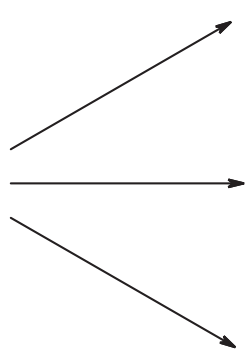

Fig. 6. Major metabolites of (A) AZD5069 and (B) AZD4721 after hepatocyte incubations in rats, dogs, and humans and in vivo from rats and dogs (see Materials and Methods). from our experiences were subsequently published (Grime and Riley, 2006).

To design a CXCR2 antagonist with improved human PK properties, we needed to both improve the bioavailability of AZD8309 and increase the effective half-life. The former was achieved by designing a monocyclic series of sulfamides with increased solubility (Austin et al., 2015). The latter was tackled by examining the data presented in Fig. 2A, with the striking observation that a compound could have high metabolic instability and yet low CL in vivo if the PPB was high enough. Since PPB appeared to have little relationship to distribution volume (Fig. 2B), this indicated that half-life could possibly be extended by raising $\mathrm{PPB}$.

Theoretically, increasing binding to blood components should reduce distribution of a drug into body tissues (Smith et al., 2010), but this was not observed in the data presented in Fig. 2B, most likely due to a combination of poor distribution properties for these acidic molecules and lipophilicity playing a "canceling out" role through impacting both PPB and affinity for tissue. Given the observation that increasing acidity is related to increasing potency and PPB (Austin et al., 2015), the proposed strategy in the CXCR 2 program was to increase acidity to increase both parameters while reducing $\log \mathrm{D}_{7.4}$ to obtain better metabolic stability. Using a CL prediction method incorporating drugbinding terms and a regression line method (Grime and Riley, 2006; Sohlenius-Sternbeck et al., 2012), a simple simulation demonstrated the general PK strategy for the program (Fig. 3).

Eventually this approach led to the discovery of AZD5069 (Fig. 1), with the substitution of nitrogen-linked for oxygen-linked pyrimidine playing a major part in giving acceptable lipophilicity, increased acidity, and the desired combination of potency, PPB, solubility, and metabolic stability (Cheshire et al., 2010; Nicholls et al., 2015). The use of an azetidine proved valuable in minimizing metabolism on the left-hand side of the molecule, as others have found (Obach et al., 2016); the diol 


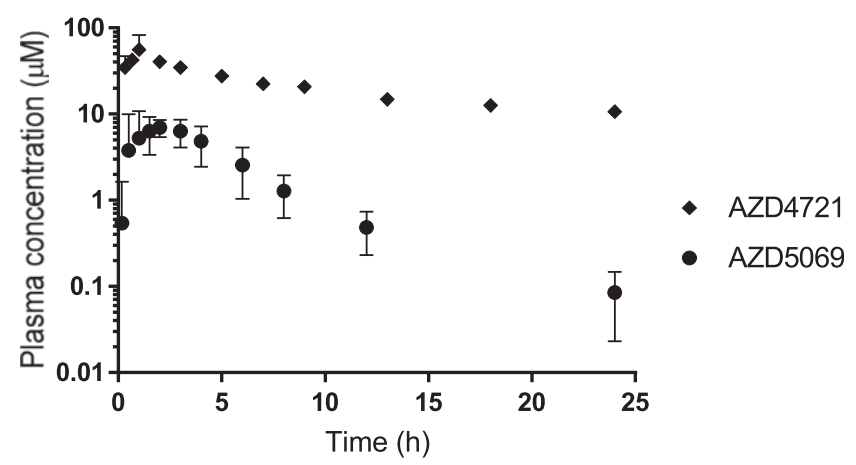

Fig. 7. Human plasma concentration (mean \pm S.D.) 24-hour time-profile after oral administration of $120 \mathrm{mg}$ of AZD5069 (O), $n=6$; or $150 \mathrm{mg}$ of AZD4721 $(\bullet), n=5$

provided both reduced $\log \mathrm{D}_{7.4}$ and reduced $\mathrm{CL}_{\text {int }}$ compared with the equivalent mono-alcohols.

AZD5069 had a short half-life in rats and dogs but with CL apparently driven solely by metabolism (Table 3 ). However, with good in vitro-in vivo extrapolation (IVIVE) for unbound hepatic metabolic $\mathrm{CL}_{\mathrm{int}}$ (in vivo/in vitro unbound $\mathrm{CL}_{\text {int }}$ ratios of between 0.76 and 1.1 for rats and dogs; Table 3 ), there was confidence in achieving the 7.5 -hour predicted human effective half-life.

Consensus opinion has converged on a definition for accuracy of clinical PK parameter prediction being observed/predicted within 2-fold (Lavé et al., 2009). For AZD5069, the 2-fold overprediction of half-life may not technically be considered failure; however, in the context of a 7.5-hour predicted half-life being realized as a 4-hour half-life, it represented a complication to the program because once-daily dosing was a primary objective. The fact that the effective half-life of AZD5069 after intravenous dosing was only 1 hour in rat and dog PK studies and human half-life was predicted to be almost 8 times longer but was in fact only 4 times longer after PO dosing prompts useful reflection on the value of allometry-based predictions.

It has been noted previously that while standard allometric scaling approaches offer fairly poor predictivity of human CL (Caldwell et al., 2004; Ward and Smith, 2004), the work of Ward and Smith highlights that human effective half-life often approximates to 4 times rat half-life, offering a useful validation of IVIVE-based predictions that should be investigated thoroughly before clinical drug development (Grime et al., 2013). Considerations of the pros and cons of allometric approaches are discussed in detail elsewhere (Di et al., 2013), but discrepancies between allometric and IVIVE predictions can come from species differences in PPB, drug-metabolizing enzyme, or drug transporter kinetics. As with all candidate drugs at AstraZeneca, the checks used for AZD5069 included an assessment linearity of in vitro intrinsic clearance with respect to drug concentration, based on the work of Obach and ReedHagen (2002); for human hepatic microsomal and hepatocyte intrinsic clearance, the data gave consistent predictions of hepatic clearance.

In attempting to understand what may have caused the discrepancy between predicted and observed effective human PK half-life, attention was drawn to the larger $V_{\mathrm{ss}}$ in dogs compared with rats and the longer terminal half-life in comparison with the effective half-life in the dog PK profile (Fig. 4). This PK profile feature is quite unusual for an acidic drug with inherently poor distribution properties. A BDC study in dogs revealed the PK profile to have a lower exposure and shorter observed terminal phase than from the noncannulated intravenous PK experiment, indicating possible enterohepatic recirculation. However, AZD5069 biliary clearance was measured as being similarly low $(0.1$ and $0.1 \mathrm{ml} / \mathrm{min}$ per kilogram, representing $1.5 \%$ and $2 \%$ of total CL) in rats and dogs, respectively. Metabolite identification data from in vitro (dog hepatocyte incubations) and in vivo samples (urine and bile analysis from the intravenous dosed dog BDC experiment) highlighted direct glucuronidation of the secondary alcohol as the major metabolite seen in dogs. In comparison, the glucuronide metabolite was less prominent in the rat in vivo, or rat and human in vitro samples for which oxidation (of the alcohols, at the $S$-benzyl position and on the azetidine ring) were also observed as principal metabolites (Fig. 6). Taken together, these data suggest that enterohepatic recirculation, involving biliary excretion of hepatically formed $O$-glucuronide metabolites followed by intestinal hydrolysis to the aglycone parent AZD5069 with subsequent reabsorption, may have extended the observable terminal PK phase in the standard intravenous dog PK experiment, leading to overestimation of predicted $V_{\mathrm{ss}}$ and effective half-life, as has been observed by others (Ouellet and Pollack, 1995; Deng et al., 2012).

Despite AZD5069 being a very attractive drug for twice-daily dosing, we still had an ambition to design a once-daily drug. There was still belief in the strategy of raising human PPB to improve half-life. Thus, through subtle structural changes AZD4721 $N$-(6-\{[(2R,3S)-3,4-dihydroxy-2butanyl]oxy\}-2-[(4-fluorobenzyl)sulfanyl]-4-pyrimidinyl)-3-methyl1-azetidinesulfonamide; (Fig. 1) was designed. The changes that achieved these improvements were methylation of the azetidine and replacement of the 2,3-difluorobenzyl with a 4-fluorobenzyl group. In fact, an increase in PPB giving a decrease in blood free fraction of almost 6-fold was achieved for AZD4721 in comparison with AZD5069, despite almost identical $\log \mathrm{D}_{7.4}$ and $\mathrm{p} K_{\mathrm{a}}$ values (Table 3). Metabolic stability was also slightly improved, with a mean human liver microsomes $\mathrm{CL}_{\text {int }}$ value approaching 2-fold lower for AZD4721.

AZD4721 was also primarily metabolized by direct glucuronidation in dogs, with a qualitatively very similar metabolic map to that of AZD5069 in all species. However, with lower $\mathrm{CL}_{\text {int }}$ and greater than 10-fold lower in vivo CL, enterohepatic recirculation was not observed in the AZD4721 dog PK profile in the sampled interval, so it had no impact on estimated $V_{\mathrm{ss}}$. Given the uncertainty around the predicted and observed human $V_{\mathrm{ss}}$ for AZD5069, as discussed earlier, $V_{\mathrm{ss}}$ for AZD4721 was set to the lower limiting value of $0.11 / \mathrm{kg}$ (Rowland and Tozer, 1989) in making the predictions of human PK.

Clinical data for AZD4721 confirmed that the strategy of increasing plasma protein binding for acidic drugs can be successful in reducing clearance to very low levels and therefore providing an effective half-life long enough for once-daily dosing with minimal $C_{\max } / C_{\min }$, despite very low distribution volume (Fig. 7). An alternative or complimentary approach can be to increase the blood-plasma ratio through drug binding to erythrocyte components. One example of this is to use the affinity of primary sulfonamides for the carbonic anhydrase enzyme (Boddy et al., 1989). Of course, for such a molecule to be an effective therapeutic drug requires at least that the pharmacologic potency is high enough at a deliverable dosage to overcome the issue of low free fraction in blood. The in vitro potency properties and sustained clinical profile of AZD4721 indicated that this should not be an issue in this case. Even though the observed halflife was 1.6-fold less than predicted, the fact that the predicted half-life approximated to the required dose interval minimized the relevance of this.

In summary, this work details the optimization strategy of acidic CXCR2 antagonists, resulting in two molecules, AZD5069 and AZD4721, suitable for twice-daily and once-daily oral dosing, respectively. This shows that under certain circumstances optimization of binding to plasma proteins can be a viable strategy to optimize effective half-life in humans.

\section{Acknowledgments}

The authors thank: Steve Connolly, Alison Wilby, Rob Riley, Jane Kenny, Anthony Atkinson, Premji Meghani, Jeffrey Stonehouse, Roger Bonnert, David 
Cheshire, Iain Walters, Mark Ebden, Alasdair Gaw, Andy Davis, Rupert Austin, Marie Cullberg, Bengt Larsson, Heather Wray, and Anna Malmgren.

\section{Authorship Contributions}

Participated in research design: Gardiner, Cox, Grime.

Conducted experiments: Gardiner, Cox, Grime.

Performed data analysis: Gardiner; Cox, Grime.

Wrote or contributed to the writing of the manuscript: Gardiner, Cox, Grime.

\section{References}

Austin R, Baxter A, Bonnert R, Hunt F, Kinchin E, and Willis P (2000) inventors, AstraZeneca AB, assignee. Novel thiazolopyrimidine compounds. Patent WO2000009511A1. 2000 Feb 24

Austin RP, Bennion C, Bonnert RV, Cheema L, Cook AR, Cox RJ, Ebden MR, Gaw A, Grime K, Meghani P, et al. (2015) Discovery and evaluation of a novel monocyclic series of CXCR2 antagonists. Bioorg Med Chem Lett 25:1616-1620.

Beeh KM, Kornmann O, Buhl R, Culpitt SV, Giembycz MA, and Barnes PJ (2003) Neutrophil chemotactic activity of sputum from patients with COPD: role of interleukin 8 and leukotriene B4. Chest 123:1240-1247.

Boddy A, Edwards P, and Rowland M (1989) Binding of sulfonamides to carbonic anhydrase: influence on distribution within blood and on pharmacokinetics. Pharm Res 6:203-209.

Bonn B, Svanberg P, Janefeldt A, Hultman Ia, and Grime K (2016) Determination of human hepatocyte intrinsic clearance for slowly metabolized compounds: comparison of a primary hepatocyte/stromal cell co-culture with plated primary hepatocytes and HepaRG. Drug Metab Dispos 44:527-533.

Bonnert RV (2004) inventor, AstraZeneca AB, assignee. Novel compound. WIPO patent WO2004026880 A1. 2004 Apr 1.

Brough SJ and McInally T (2005) inventors, AstraZeneca AB, assignee. Novel compound. U.S. patent US20050272750A1. 2005 Dec 8.

Caldwell GW, Masucci JA, Yan Z, and Hageman W (2004) Allometric scaling of pharmacokinetic parameters in drug discovery: can human CL, Vss and $\mathrm{t} 1 / 2$ be predicted from in-vivo rat data? Eur J Drug Metab Pharmacokinet 29(2):133-43.

Carlile DJ, Hakooz N, Bayliss MK, and Houston JB (1999) Microsomal prediction of in vivo clearance of CYP2C9 substrates in humans. Br J Clin Pharmacol 47:625-635.

Cheshire DR, Cox RJ, Meghani P, Preston CF, Smith NM, and Stonehouse JP (2006) inventors, Astrazeneca $\mathrm{AB}$, assignee. Pyrimidine sulphonamide derivatives as chemokine receptor modulators. WIPO patent WO2006024823A1. 2006 Mar 9.

Cheshire DR, Cox RJ, Meghani P, and Stonehouse JP (2010) inventors, AstraZeneca AB, assignee. Pyrimidine sulphonamide derivatives as chemokine receptor modulators. U.S. patent US7838675B2 2010 Nov 23.

Connolly S, Ebden MR, Langer T, Steven AR, Stewart CR, Tomlin PM, Walters IAS, and Williams AJ (2013), inventors, AstraZeneca $\mathrm{AB}$, assignee. $N$-(6- $((2 R, 3 S)-3,4$-dihydroxybutan2-yloxy)-2-(4-fluorobenzylthio)pyrimidin-4-yl)-3-methylazetidine-1-sulfonamide as chemokine receptor modulator. WIPO patent WO2013008002A1. 2013 Jan 17.

Cook D, Brown D, Alexander R, March R, Morgan P, Satterthwaite G, and Pangalos MN (2014) Lessons learned from the fate of AstraZeneca's drug pipeline: a five-dimensional framework. Nat Rev Drug Discov 13:419-431.

Cullberg M, Arfvidsson C, Larsson B, Malmgren A, Mitchell P, Wählby Hamrén U, and Wray H (2018) Pharmacokinetics of the oral selective CXCR2 antagonist AZD5069: a summary of eight phase I studies in healthy volunteers. Drugs $R$ D 18:149-159.

Davis AM and Riley RJ (2004) Predictive ADMET studies, the challenges and the opportunities. Curr Opin Chem Biol 8:378-386.

Deng J, Zhuangy X, Shen G, Lin H, and Gong Z (2012) Biliary excretion and enterohepatic circulation of thienorphine and its glucuronide conjugate in rats. Acta Pharm Sin B 2:174-180.

Di L, Feng B, Goosen TC, Lai Y, Steyn SJ, Varma MV, and Obach RS (2013) A perspective on the prediction of drug pharmacokinetics and disposition in drug research and development. Drug Metab Dispos 41:1975-1993.

Ebden MR, Meghani P, Bennion C, Cook AR, and Bonnert RV (2004) inventors, AstraZeneca AB, assignee. Pyrimidyl sulphone amide derivatives as chemokine receptor modulators. WIPO paten WO2004011443A1. 2004 Feb 5.

Fessey RE, Austin RP, Barton P, Davis AM, and Wenlock MC (2006) The role of plasma protein binding in drug discovery, in Pharmacokinetic Profiling in Drug Research: Biological, Physicochemical, and Computational Strategies (Testa B, Kramer SD, Wunderli-Allenspach H, and Folkers G eds) pp 119-141, Wiley-VCH, Weinheim, Baden-Württemberg.

Gardiner P and Paine SW (2011) The impact of hepatic uptake on the pharmacokinetics of organic anions. Drug Metab Dispos 39:1930-1938.

Grime K and Riley RJ (2006) The impact of in vitro binding on in vitro-in vivo extrapolations, projections of metabolic clearance and clinical drug-drug interactions. Curr Drug Metab 7:251-264.

Grime KH, Barton P, and McGinnity DF (2013) Application of in silico, in vitro and preclinical pharmacokinetic data for the effective and efficient prediction of human pharmacokinetics. $\mathrm{Mol}$ Pharm 10:1191-1206.

Grover A and Benet LZ (2009) Effects of drug transporters on volume of distribution. AAPS J 11: 250-261.

Highfill SL, Cui Y, Giles AJ, Smith JP, Zhang H, Morse E, Kaplan RN, and Mackall CL (2014) Disruption of CXCR2-mediated MDSC tumor trafficking enhances anti-PD1 efficacy. Sci Transl Med 6:237ra67.

Holmes WE, Lee J, Kuang WJ, Rice GC, and Wood WI (1991) Structure and functional expression of a human interleukin-8 receptor. Science 253:1278-1280.

Houston JB (2013) Prediction of human pharmacokinetics in 2013 and beyond. Drug Metab Dispos 41:1973-1974.

Houston JB and Carlile DJ (1997) Prediction of hepatic clearance from microsomes, hepatocytes, and liver slices. Drug Metab Rev 29:891-922.
Ito K and Houston JB (2005) Prediction of human drug clearance from in vitro and preclinical data using physiologically based and empirical approaches. Pharm Res 22:103-112.

Iwatsubo T, Hirota N, Ooie T, Suzuki H, Shimada N, Chiba K, Ishizaki T, Green CE, Tyson CA and Sugiyama Y (1997) Prediction of in vivo drug metabolism in the human liver from in vitro metabolism data. Pharmacol Ther 73:147-171.

Jamieson T, Clarke M, Steele CW, Samuel MS, Neumann J, Jung A, Huels D, Olson MF, Das S, Nibbs RJ, et al. (2012) Inhibition of CXCR2 profoundly suppresses inflammation-driven and spontaneous tumorigenesis. J Clin Invest 122:3127-3144.

Kissinger JT, Garver EM, Schnell MA, Schantz JD, Coatney RW, and Meunier LD (1998) A new method to collect bile and access the duodenum in conscious dogs. Contemp Top Lab Anim Sci 37:89-93.

Lavé T, Chapman K, Goldsmith P, and Rowland M (2009) Human clearance prediction: shifting the paradigm. Expert Opin Drug Metab Toxicol 5:1039-1048.

Leaker BR, Barnes PJ, and O'Connor B (2013) Inhibition of LPS-induced airway neutrophilic inflammation in healthy volunteers with an oral CXCR2 antagonist. Respir Res 14:137.

Lombardo F, Obach RS, Varma MV, Stringer R, and Berellini G (2014) Clearance mechanism assignment and total clearance prediction in human based upon in silico models. J Med Chem 57: $4397-4405$.

McGinnity DF, Soars MG, Urbanowicz RA, and Riley RJ (2004) Evaluation of fresh and cryopreserved hepatocytes as in vitro drug metabolism tools for the prediction of metabolic clearance. Drug Metab Dispos 32:1247-1253.

Mukaida N (2003) Pathophysiological roles of interleukin-8/CXCL8 in pulmonary diseases. Am J Physiol Lung Cell Mol Physiol 284:L566-L577.

Murphy PM and Tiffany HL (1991) Cloning of complementary DNA encoding a functional human interleukin-8 receptor. Science 253:1280-1283.

Nicholls DJ, Wiley K, Dainty I, MacIntosh F, Phillips C, Gaw A, and Mårdh CK (2015) Pharmacological characterization of AZD5069, a slowly reversible CXC chemokine receptor 2 antagonist. J Pharmacol Exp Ther 353:340-350.

Obach RS, LaChapelle EA, Brodney MA, Vanase-Frawley M, Kauffman GW, and Sawant-Basak A (2016) Strategies toward optimization of the metabolism of a series of serotonin-4 partial agonists: investigation of azetidines as piperidine isosteres. Xenobiotica 46:1112-1121.

Obach RS and Reed-Hagen AE (2002) Measurement of Michaelis constants for cytochrome P450mediated biotransformation reactions using a substrate depletion approach. Drug Metab Dispos 30:831-837.

Ouellet DM and Pollack GM (1995) Biliary excretion and enterohepatic recirculation of morphine3-glucuronide in rats. Drug Metab Dispos 23:478-484.

Pang KS and Rowland M (1977) Hepatic clearance of drugs. I. Theoretical considerations of a "well-stirred" model and a "parallel tube" model. Influence of hepatic blood flow, plasma and blood cell binding, and the hepatocellular enzymatic activity on hepatic drug clearance. I Pharmacokinet Biopharm 5:625-653.

Riley RJ, Martin IJ, and Cooper AE (2002) The influence of DMPK as an integrated partner in modern drug discovery. Curr Drug Metab 3:527-550.

Rowland M and Tozer TN(1989) Clinical Pharmacokinetics: Concepts and Applications, 3rd ed, Williams \& Wilkins, Philadelphia.

Schuster D, Laggner C, and Langer T (2005) Why drugs fail--a study on side effects in new chemical entities. Curr Pharm Des 11:3545-3559.

Seglen PO (1976) Preparation of isolated rat liver cells. Methods Cell Biol 13:29-83.

Smith DA, Di L, and Kerns EH (2010) The effect of plasma protein binding on in vivo efficacy: misconceptions in drug discovery. Nat Rev Drug Discov 9:929-939.

Soars MG, Grime K, Sproston JL, Webborn PJ, and Riley RJ (2007) Use of hepatocytes to asses the contribution of hepatic uptake to clearance in vivo. Drug Metab Dispos 35:859-865.

Sohlenius-Sternbeck A-K, Jones C, Ferguson D, Middleton BJ, Projean D, Floby E, Bylund J, and Afzelius L (2012) Practical use of the regression offset approach for the prediction of in vivo intrinsic clearance from hepatocytes. Xenobiotica 42:841-853.

Steele CW, Karim SA, Leach JDG, Bailey P, Upstill-Goddard R, Rishi L, Foth M, Bryson S, McDaid K, Wilson Z, et al. (2016) CXCR2 inhibition profoundly suppresses metastases and augments immunotherapy in pancreatic ductal adenocarcinoma. Cancer Cell 29:832-845.

Virtala R, Ekman A-K, Jansson L, Westin U, and Cardell LO (2012) Airway inflammation evaluated in a human nasal lipopolysaccharide challenge model by investigating the effect of a CXCR2 inhibitor. Clin Exp Allergy 42:590-596.

Walters I, Austin C, Austin R, Bonnert R, Cage P, Christie M, Ebden M, Gardiner S, Grahames C Hill S, et al. (2008) Evaluation of a series of bicyclic CXCR2 antagonists. Bioorg Med Chem Lett 18:798-803.

Ward KW and Smith BR (2004) A comprehensive quantitative and qualitative evaluation of extrapolation of intravenous pharmacokinetic parameters from rat, dog, and monkey to humans. I. Clearance. Drug Metab Dispos 32(6):603-11.

Waring MJ, Arrowsmith J, Leach AR, Leeson PD, Mandrell S, Owen RM, Pairaudeau G, Pennie WD, Pickett SD, Wang J, et al. (2015) An analysis of the attrition of drug candidates from four major pharmaceutical companies. Nat Rev Drug Discov 14:475-486.

Wenlock MC, Barton P, and Austin RP (2011a) A kinetic method for the determination of plasma protein binding of compounds unstable in plasma: specific application to enalapril. $J$ Pharm Biomed Anal 55:385-390.

Wenlock MC, Austin RP, Potter T, and Barton P (2011b) A highly automated assay for determining the aqueous equilibrium solubility of drug discovery compounds. J Lab Autom 16: 276-284.

Willis PA, Bonnert RV, Hunt SF, and Walters IAS (2001) inventors, AstraZeneca UK Limited, assignee. Novel thiazolo(4,5-d)pyrimidine compounds. WIPO patent WO200125242A1. 2001 Apr 12 .

Address correspondence to: Philip Gardiner, CPSS, R\&D BioPharmaceuticals, AstraZeneca, Pepparedsleden 1, SE-431 83 Mölndal, Sweden. E-mail: philip.gardiner@ astrazeneca.com 